\title{
Non-Markovian finite-temperature two-time correlation functions of system operators: beyond the quantum regression theorem
}

\author{
Hsi-Sheng Goan, $, 1,2$, 田 Po-Wen Chen, ${ }^{1,2}$ and Chung-Chin Jian ${ }^{1,2}$ \\ ${ }^{1}$ Department of Physics and Center for Theoretical Sciences, \\ National Taiwan University, Taipei 10617, Taiwan \\ ${ }^{2}$ Center for Quantum Science and Engineering, National Taiwan University, Taipei 10617, Taiwan
}

\begin{abstract}
An extremely useful evolution equation that allows systematically calculating the two-time correlation functions (CF's) of system operators for non-Markovian open (dissipative) quantum systems is derived. The derivation is based on perturbative quantum master equation approach, so nonMarkovian open quantum system models that are not exactly solvable can use our derived evolution equation to easily obtain their two-time CF's of system operators, valid to second order in the system-environment interaction. Since the form and nature of the Hamiltonian are not specified in our derived evolution equation, our evolution equation is applicable for bosonic and/or fermionic environments and can be applied to a wide range of system-environment models with any factorized (separable) system-environment initial states (pure or mixed). When applied to a general model of a system coupled to a finite-temperature bosonic environment with a system coupling operator $L$ in the system-environment interaction Hamiltonian, the resultant evolution equation is valid for both $L=L^{\dagger}$ and $L \neq L^{\dagger}$ cases, in contrast to those evolution equations valid only for $L=L^{\dagger}$ case in the literature. The derived equation that generalizes the quantum regression theorem (QRT) to the non-Markovian case will have broad applications in many different branches of physics. We then give conditions on which the QRT holds in the weak system-environment coupling case, and apply the derived evolution equation to a problem of a two-level system (atom) coupled to a finite-temperature bosonic environment (electromagnetic fields) with $L \neq L^{\dagger}$.
\end{abstract}

PACS numbers: 03.65.Ca, 03.65.Yz, 05.30.-d, 42.50.Lc

\section{INTRODUCTION}

In many fields of modern sciences, one has to deal with open quantum systems in contact with their quantum surroundings or environments (reservoirs or baths) [1 9]. Most often, one concerns with only the system dynamics and the key quantity is the reduced system density matrix $\rho(t)$ defined as the partial trace of the total systemplus-reservoir density operator $\rho_{T}(t)$ over the reservoir degrees of freedom; i.e., $\rho(t)=\operatorname{Tr}_{R}\left[\rho_{T}(t)\right]$. The evolution equation of the reduced density matrix is governed by the reduced Liouville equation or called the quantum master equation that can be Markovian or non-Markovian.

Two-time (multi-time) correlation functions (CF's) of an open quantum system are important physical quantities. They can provide significant information about the system, whereas the single-time expectation values can not. For example, the two-time CF's of the electromagnetic field emitted by an atom are required for calculating the fluorescence spectrum [1-5]. The two-time CF's of the number of emitted photons give the information about the photon statistics and describe the behavior of photon bunching and anti-bunching $[1-5]$. The two-time CF's of the electric current through nanostructure devices are useful in the study of the transport properties of current fluctuations and noise spectrum [10, 11]. In the Markovian case, an extremely useful procedure to

\footnotetext{
* Corresponding author: goan@phys.ntu.edu.tw
}

calculate the two-time (multi-time) CF's for open (dissipative) quantum systems is the so-called quantum regression theorem (QRT) [1-5] that gives a direct relation between the time evolution equation of the single-time expectation values and that of their corresponding twotime (multi-time) CF's. So knowing the time evolution of the reduced density matrix of the system allows one to calculate all of the single-time expectation values and two-time (multi-time) CF's in the Markovian case. This is, however, no longer true in the non-Markovian case. For non-Markovian open (dissipative) quantum systems, the QRT is not valid in general [8, 9, 12 17]. Although it is commendable to use the exact procedures [8, 9, 14, 15] to calculate directly the non-Markovian two-time CF's, not too many problems can be exactly worked out in this way. It is thus important that a procedure similar to the QRT can be developed and be applied to calculate the two-time CF's perturbatively for the non-Markovian open (dissipative) quantum systems.

Recently, by using the stochastic Schrödinger equation approach and the Heisenberg equation of system operator method, Alonso and de Vega derived [18 20] the evolution equations of the two-time (multi-time) CF's of the system operators for a general model of a quantum system coupled to a bosonic environment with a system coupling operator $L$ in the system-environment interaction Hamiltonian. The evolution equations, valid to second order in system-environment coupling strength, were applied to calculate the emission spectra of a two-level atom placed in a structured non-Markovian environment, i.e, electromagnetic fields in a photonic band-gap mate- 
rial [21]. In the photonic band-gap material, the correlation function of the electromagnetic field (environment) is highly non-Markovian, particularly within the edges of the bands, so the QRT is not valid even when the atom-environment interaction strength is weak. Although the evolution equations, derived in Refs. [18 20] (Eq. (6) in Ref. 18], Eq. (31) in Ref. [19] and Eq. (60) in Ref. 20]), are very useful to calculate the time evolution of the two-time (multi-time) CF's of the system observables, the derivations of the evolution equations were presented only for an environment at zero temperature and only for a system state in an initial pure state. In Ref. [19], it was mentioned that it is possible to use the reduced stochastic system propagator that corresponds to an initial state of the environment different from the vacuum to evaluate the single-time expectation values and multi-time CF's with more general initial conditions. But they derived only a master equation that is conditioned on initial bath states and is capable of evaluating just the single-time expectation values of system observables for general initial conditions, both for an initial pure state and mixed state [19]. So strictly speaking, the two-time evolution equations derived in Refs. [18 20] is applicable only for a zero-temperature environment. However, these equations were used to calculate the two-time CF's of system observables of dissipative spin-boson models at finite temperatures [18 20]. This is possible only for the dissipative spin-boson models with Hermitian system coupling operators, $L=L^{\dagger}$ (see also the discussions in subsection ஹI A).

In this paper, we use another commonly used technique, the quantum master equation approach [1-7], to derive in the weak system-environment coupling limit an evolution equation of the two-time CF's of the system operators for non-Markovian open quantum systems in finite-temperature environments for any factorized (separable) system-environment initial states (pure or mixed). This quantum master equation approach, different from those in Refs. [18 20], allows us to explicitly point out an important nonlocal environment (bath) memory term that vanishes in the Markovian case but makes the evolution equation deviate from the QRT in general cases. Since the form and nature of the Hamiltonian are not specified in our derived evolution equation, the evolution equation is applicable for bosonic and/or fermionic environments and can be applied to a wider range of systemenvironment models. When applied to a general model of a system coupled to a finite-temperature bosonic environment, our resultant two-time evolution equation is valid for both Hermitian and non-Hermitian system coupling operator cases. This is in contrast to the two-time evolution equations derived in Refs. [18 20] valid only for the finite-temperature bosonic environment case with a Hermitian system coupling operator $L=L^{\dagger}$. Our derived equation that generalizes the QRT to the nonMarkovian case will have broad applications in many different branches of physics.

The paper is organized as follows. In Sec. II we derive the non-Markovian finite-temperature evolution equations for one-time expectation values and two-time CF's for a general system-environment thermal model. We then apply the derived equations (valid to second order in the system-environment interaction) to a thermal bosonic environment and work out the explicit forms of the evolution equations in terms of system coupling operators in Sec. III] The evolution equations for a nonMarkovian bosonic environment has been presented in Ref. [22] without any derivation. In this paper, the detailed derivation is given. We also discuss the connection of our derived two-time evolution equations with those presented in Refs. [18 20] and give conditions on which the QRT may hold in the weak system-environment coupling case. In Sec. IV] we apply the newly derived equation to a problem of a two-level system (atom) coupled to a bosonic environment (electromagnetic fields), in which the system coupling operator is non-Hermitian, i.e., $L \neq L^{\dagger}$. A short conclusion is given in Sec. $\mathrm{V}$

\section{DERIVATIONS OF EVOLUTION EQUATIONS}

\section{A. Time-nonlocal and time-local quantum master equations}

Let us consider a general total Hamiltonian of the system and reservoir (environment) as

$$
H=H_{S}+H_{I}+H_{R},
$$

where $H_{S}$ and $H_{R}$ are the Hamiltonian for system and reservoir, respectively, and $H_{I}$ is the interaction between the system and reservoir. It is convenient to go to the interaction picture in which

$$
d \tilde{\rho}_{T}(t) / d t=-(i / \hbar)\left[\tilde{H}_{I}(t), \tilde{\rho}_{T}(t)\right],
$$

where $\tilde{H}_{I}(t)=e^{i H_{0} t / \hbar} H_{I} e^{-i H_{0} t / \hbar}$ is explicitly timedependent, $\tilde{\rho}_{T}(t)=e^{i H_{0} t / \hbar} \rho_{T}(t) e^{-i H_{0} t / \hbar}$ is the total density matrix operator at time $t$ in the interaction picture and $H_{0}=H_{S}+H_{R}$. Then the Liouville equation of the total density matrix $\rho_{T}(t)$ in the Schrödinger picture becomes

$$
\frac{d \rho_{T}(t)}{d t}=-\frac{i}{\hbar}\left[H_{0}, \rho_{T}(t)\right]+e^{-i H_{0} t / \hbar} \frac{d \tilde{\rho}_{T}(t)}{d t} e^{i H_{0} t / \hbar} .
$$

One can integrate Eq. (2) formally to obtain

$$
\tilde{\rho}_{T}(t)=\tilde{\rho}_{T}(0)-\frac{i}{\hbar} \int_{0}^{t} d t^{\prime}\left[\tilde{H}_{I}\left(t^{\prime}\right), \tilde{\rho}_{T}\left(t^{\prime}\right)\right]
$$

and substitute for $\tilde{\rho}_{T}(t)$ inside the commutator in Eq. (2). The resultant equation is

$$
\begin{aligned}
\frac{d \tilde{\rho}_{T}(t)}{d t}= & -\frac{i}{\hbar}\left[\tilde{H}_{I}(t), \tilde{\rho}_{T}(0)\right] \\
& -\frac{1}{\hbar^{2}} \int_{0}^{t} d t^{\prime}\left[\tilde{H}_{I}(t),\left[\tilde{H}_{I}\left(t^{\prime}\right), \tilde{\rho}_{T}\left(t^{\prime}\right)\right]\right] .
\end{aligned}
$$


Equation (5) is still exact. Suppose initially $\rho_{T}(0)=\tilde{\rho}_{T}(0)=\rho(0) \otimes R_{0}$, where $R_{0}=$ $\exp \left(-H_{R} / k_{B} T\right) / \operatorname{Tr}_{R}\left[\exp \left(-H_{R} / k_{B} T\right)\right]$ is the initial thermal reservoir density operator. Then after tracing over the reservoir, Eq. (5) gives the master equation of the reduced density matrix in the interaction picture

$$
\frac{d \tilde{\rho}(t)}{d t}=-\frac{1}{\hbar^{2}} \operatorname{Tr}_{R} \int_{0}^{t} d t^{\prime}\left[\tilde{H}_{I}(t),\left[\tilde{H}_{I}\left(t^{\prime}\right), \tilde{\rho}_{T}\left(t^{\prime}\right)\right]\right],(6)
$$

where we have considered a class of system-reservoir interaction Hamiltonian models for the thermal reservoir such that

$$
\operatorname{Tr}_{R}\left[\tilde{H}_{I}(t) R_{0}\right]=0
$$

to eliminate the first term in Eq. (5).

The perturbative non-Markovian open quantum system theory may be categorized into two classes: the time-nonlocal (or time-convolution) $] 7,24-29$ and the time-local (or time-convolutionless) [6, 7, 25-36] methods. This has been discussed extensively in the literature [7, 25, 28, 29]. The environment or reservoir by definition is large and contains many degrees of freedom so that the influence of the system on the reservoir is small in the weak system-environment coupling case. As a consequense, to second order in system-environment interaction, the total density operator on the right hand side of Eq. (6) can be approximated to an uncorrelated (factorized) state as $\tilde{\rho}_{T}\left(t^{\prime}\right)=\tilde{\rho}\left(t^{\prime}\right) \otimes R_{0}+\mathcal{O}\left(\tilde{H}_{I}\right)$ [2] since the products of two interaction Hamiltonians $\tilde{H}_{I}$ 's appear already there. So in many textbooks [1-3], the replacement of $\tilde{\rho}_{T}\left(t^{\prime}\right)$ with $\tilde{\rho}\left(t^{\prime}\right) \otimes R_{0}$ in Eq. (6) is performed under the so-called Born approximation. One then obtains [13, 7, 24, 29]

$$
\frac{d \tilde{\rho}(t)}{d t}=-\frac{1}{\hbar^{2}} \operatorname{Tr}_{R} \int_{0}^{t} d t^{\prime}\left[\tilde{H}_{I}(t),\left[\tilde{H}_{I}\left(t^{\prime}\right), \tilde{\rho}\left(t^{\prime}\right) \otimes R_{0}\right]\right] .
$$

Note that Eq. (8) is in a form of delayed integrodifferential equation and is thus a time-nonlocal master equation. However, it can also be shown that another systematically perturbative non-Markovian master equation that is local in time [6, 7, 25 36] can be derived from the time-convolutionless projection operator formalism [7, 25 28, 30] or from the iteration expansion method [ [6]. Under the similar assumption of the factorized initial system-reservoir density matrix state and the use of Eq. (7), the second-order time-convolutionless master equation in the interaction picture can be obtained as [6, 7, 25,36$]$

$$
\frac{d \tilde{\rho}(t)}{d t}=-\frac{1}{\hbar^{2}} \operatorname{Tr}_{R} \int_{0}^{t} d t^{\prime}\left[\tilde{H}_{I}(t),\left[\tilde{H}_{I}\left(t^{\prime}\right), \tilde{\rho}(t) \otimes R_{0}\right]\right] .
$$

We note here that obtaining the time-convolutionless non-Markovian master equation perturbatively up to only second order in the interaction Hamiltonian using the time-convolutionless projection operator technique 7, 25, 26] is equivalent to obtaining it by replacing $\tilde{\rho}\left(t^{\prime}\right)$ with $\tilde{\rho}(t)$ in Eq. (8) [6, 7, 25 36]. One may be tempted to think that the second-order time-nonlocal master equation (8) is more accurate than the second-order time-local (time-convolutionless) master equation (9) since besides the Born approximation, the (first) Markovian approximation of replacing $\tilde{\rho}\left(t^{\prime}\right)$ with $\tilde{\rho}(t)$ in Eq. (8) seems to be an additional approximation made on the timelocal master equation. But it was shown that this may not be the case. In many examples [7, 25 27, 29, 31], the time-convolutionless approach works better than the time-nonlocal approach when the exact dynamics is used to test the perturbative non-Markovian theory based on these two approaches. Here we will consider the second-order non-Markovian time-convolutionless (timelocal) evolution equation in our derivation.

\section{B. Quantum regression procedure}

Quantum regression procedure or QRT indicates that for (Markovian) open quantum systems, the equations of motion (or evolution equations) for single-time expectation values of system operators are also the equations of motion for two-time (multi-time) CF's. Formally, the single-time or one-time expectation values of system operators can be written as

$$
\left\langle A\left(t_{1}\right)\right\rangle=\operatorname{Tr}_{S \otimes R}\left[A\left(t_{1}\right) \rho_{T}(0)\right]=\operatorname{Tr}_{S \otimes R}\left[A(0) \rho_{T}\left(t_{1}\right)\right],
$$

where $A\left(t_{1}\right)$ represents a general system Heisenberg operator(s), $\rho_{T}\left(t_{1}\right)$ is the Schrödinger total density matrix operator at time $t_{1}$, and the subscript of $S \otimes R$ attached to the trace symbol of Tr indicates a trace over the Hilbert space of the total composite system [23]. The evolution equation of the one-time expectation values of system operators can then be obtained as

$$
\begin{aligned}
d\left\langle A\left(t_{1}\right)\right\rangle / d t_{1} & =\operatorname{Tr}_{S \otimes R}\left[A\left(d \rho_{T}\left(t_{1}\right) / d t_{1}\right)\right] \\
& =\operatorname{Tr}_{S}\left[A\left(d \rho\left(t_{1}\right) / d t_{1}\right)\right],
\end{aligned}
$$

where we have used the fact that $A=A(0)$ is a pure system operator and $\operatorname{Tr}_{R}\left[d \rho_{T}\left(t_{1}\right) / d t\right]=d \rho\left(t_{1}\right) / d t$. For the two-time $\mathrm{CF}$ with $t_{1}>t_{2}$, one has

$$
\begin{aligned}
\left\langle A\left(t_{1}\right) B\left(t_{2}\right)\right\rangle & =\operatorname{Tr}_{S \otimes R}\left[A\left(t_{1}\right) B\left(t_{2}\right) \rho_{T}(0)\right] \\
& =\operatorname{Tr}_{S \otimes R}\left[A e^{-i H t / \hbar} B \rho_{T}\left(t_{2}\right) e^{i H t / \hbar}\right]
\end{aligned}
$$

where $t=t_{1}-t_{2}$, and $A=A(0)$ and $B=B(0)$ are system operators. Let $\chi_{T}(0)=B \rho_{T}\left(t_{2}\right)$. Then the two-time CF (13) becomes $\left\langle A\left(t_{1}\right) B\left(t_{2}\right)\right\rangle=\operatorname{Tr}_{S \otimes R}\left[A \chi_{T}(t)\right]$. It is then equivalent to the expectation value of the operator $A$ with respect to the effective density matrix operator $\chi_{T}(t)=e^{-i H t / \hbar} B \rho_{T}\left(t_{2}\right) e^{i H t / \hbar}$ that satisfies the same Liouville evolution equation as $\rho_{T}(t)$ even though $\chi_{T}(t)$ may not be a proper density matrix (i.e., positive-definite trace-conservative operator). The evolution equation of 
the two-time CF can be formally written as

$$
\begin{aligned}
d\left\langle A\left(t_{1}\right) B\left(t_{2}\right)\right\rangle / d t_{1} & =\operatorname{Tr}_{S \otimes R}\left[A\left(d \chi_{T}(t) / d t\right)\right] \\
& =\operatorname{Tr}_{S}[A(d \chi(t) / d t)],
\end{aligned}
$$

where the relations of the reduced operator $\chi(t)=$ $\operatorname{Tr}_{R}\left[\chi_{T}(t)\right]$ and $\operatorname{Tr}_{R}\left[d \chi_{T}(t) / d t\right]=d \chi(t) / d t$ have been used. If the reduced master equations $d \chi(t) / d t$ and $d \rho(t) / d t$ had the same operator equation form, one might conclude that the structure and the form of the evolution equation of the two-time CF would be the same as those of the single-time evolution equation and thus the QRT would apply. In fact, it has been shown that the QRT is not valid in general [14, 15], but the QRT or regression procedure is useful and correct for systems where the coupling to reservoirs is weak and the Markovian approximation holds [2, 16, 17]. The main purpose of the present paper is to derive the non-Markovian finite-temperature evolution equation of the two-time system CF's using a quantum master equation approach, an approach different from those in Refs. [19, 20]. Our equations, which are valid for both a Hermitian and a non-Hermitian system coupling operators and thus generalize the corresponding results in Refs. [19, 20], can be used to calculate the twotime CF's for any factorized (separable) system-reservoir initial state and for any arbitrary temperature as long as the approximation of the weak system-environment coupling still holds.

\section{Evolution equations in the weak system-environment coupling limit}

Let us proceed to first derive perturbatively the explicit evolution equation of the single-time expectation values in the non-Markovian case. Here we consider the second-order non-Markovian time-convolutionless (timelocal) evolution equation in our derivation. We wish to obtain an evolution equation, $d \rho_{T}(t) / d t$, valid to second order in system-environment interaction Hamiltonian, to substitute into Eq. (11) for the single-time expectation values and into Eq. (14) for the two-time CF's. It is convenient to first go to the interaction picture and obtain a time-local (time-convolutionless) evolution equation of the density matrix valid to that order. This can be achieved by the substitution of $\tilde{\rho}_{T}\left(t^{\prime}\right) \rightarrow \tilde{\rho}_{T}(t)$ in the second term on the right hand side of the equal sign of Eq. (5) [6, 7, 25 37]. To go back to the Schrödinger picture, we substitute the resultant second-order equation obtained from Eq. (5) into Eq. (3) to obtain the evolution equation, $d \rho_{T}(t) / d t$. By substituting this equation $d \rho_{T}(t) / d t$ valid to second order in the interaction Hamiltonian into Eq. (11), the evolution equation of the singletime expectation values then consists of three terms. The second term involves the first term on the right hand side of the equal sign of Eq. (5), and will vanish on the conditions that $\rho_{T}(0)=\tilde{\rho}_{T}(0)=\rho(0) \otimes R_{0}$ and $\operatorname{Tr}_{R}\left[\tilde{H}_{I}(t) R_{0}\right]=0$ [Eq. (7)] are satisfied. As a result, we obtain up to second order in the interaction Hamiltonian

$$
\begin{aligned}
\frac{d\left\langle A\left(t_{1}\right)\right\rangle}{d t_{1}}= & \frac{i}{\hbar} \operatorname{Tr}_{S \otimes R}\left(\left[H_{S}, A\right] \rho_{T}\left(t_{1}\right)\right) \\
+ & \frac{1}{\hbar^{2}} \int_{0}^{t_{1}} d \tau \operatorname{Tr}_{S \otimes R} \\
& \quad\left(\tilde{H}_{I}\left(\tau-t_{1}\right)\left[A, H_{I}\right] \rho_{T}\left(t_{1}\right)\right. \\
& \left.\quad+\left[H_{I}, A\right] \tilde{H}_{I}\left(\tau-t_{1}\right) \rho_{T}\left(t_{1}\right)\right) \\
= & (i / \hbar) \operatorname{Tr}_{S \otimes R}\left(\left\{\left[H_{S}, A\right]\right\}\left(t_{1}\right) \rho_{T}(0)\right) \\
+ & \frac{1}{\hbar^{2}} \int_{0}^{t_{1}} d \tau \operatorname{Tr}_{S \otimes R} \\
& \quad\left(\left\{\tilde{H}_{I}\left(\tau-t_{1}\right)\left[A, H_{I}\right]\right\}\left(t_{1}\right) \rho_{T}(0)\right. \\
& \left.+\left\{\left[H_{I}, A\right] \tilde{H}_{I}\left(\tau-t_{1}\right)\right\}\left(t_{1}\right) \rho_{T}(0)\right),
\end{aligned}
$$

where we have transformed from the Schrödinger picture to the Heisenberg picture in the second equal sign and $\{A B\}(t) \equiv \exp (i H t / \hbar) A B \exp (-i H t / \hbar)$.

Since $\chi_{T}(t)$ and $\rho_{T}(t)$ obey the same equations of Eqs. (3) and (5), at first sight, one may think that the two-time evolution equations, Eqs. (14) and (15), are similar to the single-time evolution equations, Eqs. (11) and (12), and thus might be tempted to conclude that they have the same form of the evolution equations. Indeed, by using Eqs. (14), (3) and (5), the first and third terms of the resultant equation derived from Eq. (14) are similar to the right-hand side of the single-time evolution equation (16) with the replacement of $\rho_{T}(0) \rightarrow \chi_{T}\left(-t_{2}\right)=$ $B\left(t_{2}\right) \rho_{T}(0)$ and with the change of the integration region from $\left[0, t_{1}\right]$ to $\left[t_{2}, t_{1}\right]$. Then we obtain

$$
\begin{aligned}
& \frac{i}{\hbar} \operatorname{Tr}_{S \otimes R}\left(\left\{\left[H_{S}, A\right]\right\}\left(t_{1}\right) B\left(t_{2}\right) \rho_{T}(0)\right) \\
& +\frac{1}{\hbar^{2}} \int_{t_{2}}^{t_{1}} d \tau \operatorname{Tr}_{S \otimes R} \\
& \quad\left(\left\{\tilde{H}_{I}\left(\tau-t_{1}\right)\left[A, H_{I}\right]\right\}\left(t_{1}\right) B\left(t_{2}\right) \rho_{T}(0)\right. \\
& \left.\quad+\left\{\left[H_{I}, A\right] \tilde{H}_{I}\left(\tau-t_{1}\right)\right\}\left(t_{1}\right) B\left(t_{2}\right) \rho_{T}(0)\right) .
\end{aligned}
$$

However, a significant difference is that the expectation values for the second term does not vanish, i.e.,

$$
(-i / \hbar) \operatorname{Tr}_{S \otimes R}\left(A e^{-i H_{0} t / \hbar}\left[\tilde{H}_{I}(t), \tilde{\chi}_{T}(0)\right] e^{i H_{0} t / \hbar}\right) \neq 0,
$$

in the non-Markovian case, where $t=t_{1}-t_{2}$ in Eq. (18). The reason can be understood as follows. The interaction Hamiltonian $\tilde{H}_{I}\left(t_{1}-t_{2}\right)$ in Eq. (18) involves the environment operators in the time interval from $t_{2}$ to $t_{1}$, and the effective density matrix operator $\tilde{\chi}_{T}(0)$ can be written as $\tilde{\chi}_{T}(0)=\chi_{T}(0)=B \rho_{T}\left(t_{2}\right)=B U\left(t_{2}, 0\right) \rho_{T}(0) U^{\dagger}\left(t_{2}, 0\right)$, where $U\left(t_{2}, 0\right)=e^{-i H t_{2} / \hbar}$ is the Heisenberg evolution operator of the total Hamiltonian from time 0 to $t_{2}$. If the environment is Markovian where the environment operator CF's at two different times are $\delta$-correlated in 
time, then we may regard that the environment operators in $\tilde{H}_{I}\left(t_{1}-t_{2}\right)$ are not correlated with those in $U\left(t_{2}, 0\right)$. So the trace over the environment degrees of freedom for operator $\tilde{H}_{I}\left(t_{1}-t_{2}\right)$ and operator $U\left(t_{2}, 0\right)$ can be performed independently or separately. The trace of $\rho_{T}\left(t_{2}\right)=U\left(t_{2}, 0\right) \rho_{T}(0) U^{\dagger}\left(t_{2}, 0\right)$ over the environment degrees of freedom yields the reduced density matrix $\rho\left(t_{2}\right)=\operatorname{Tr}_{R}\left[\rho_{T}\left(t_{2}\right)\right]$, but the trace of $\tilde{H}_{I}\left(t_{1}-t_{2}\right)$ vanishes, i.e., $\operatorname{Tr}_{R}\left[\tilde{H}_{I}\left(t_{1}-t_{2}\right) R_{0}\right]=0$, because of Eq. (7). Thus Eq. (18) vanishes in the Markovian limit. But the situation differs for a non-Markovian environment as the environment operator in $\tilde{H}_{I}\left(t_{1}-t_{2}\right)$ may, in general, be correlated with that in $U\left(t_{2}, 0\right)$. Therefore, the evolution from $\rho_{T}(0)$ to $\rho_{T}\left(t_{2}\right)$ under the influence of interaction Hamiltonian in the presence of the reservoir needs to be taken into account before the trace over the environment is performed in Eq. (18). We emphasize here that it is this nonlocal environment (bath) memory term, Eq. (18), that vanishes in the Markovian case but makes the evolution equation of the two-time CF's of the system operators deviate from the QRT. As we aim to obtain an evolution equation of the two-time CF's of the system operators, valid up to second order in the interaction Hamiltonian, we need to find $\rho_{T}\left(t_{2}\right)$ only up to first order in the interaction Hamiltonian. So substituting $\rho_{T}\left(t_{2}\right)=e^{-i H_{0} t_{2} / \hbar} \tilde{\rho}_{T}\left(t_{2}\right) e^{i H_{0} t_{2} / \hbar}$ with the expression

$$
\tilde{\rho}_{T}\left(t_{2}\right) \approx \tilde{\rho}_{T}(0)-\frac{i}{\hbar} \int_{0}^{t_{2}} d \tau\left[\tilde{H}_{I}(\tau), \tilde{\rho}_{T}\left(t_{2}\right)\right]
$$

for $\tilde{\chi}_{T}(0)=\chi_{T}(0)=B \rho_{T}\left(t_{2}\right)$ in Eq. (18), we then obtain up to second order in the interaction Hamiltonian (in the system-environment coupling strength)

$$
\begin{aligned}
& -\frac{1}{\hbar^{2}} \int_{0}^{t_{2}} d \tau \operatorname{Tr}_{S \otimes R}\left(A e ^ { - i H _ { 0 } t / \hbar } \left[\tilde{H}_{I}(t),\right.\right. \\
& \left.\left.B e^{-i H_{0} t_{2} / \hbar}\left[\tilde{H}_{I}(\tau), \tilde{\rho}_{T}\left(t_{2}\right)\right] e^{i H_{0} t_{2} / \hbar}\right] e^{i H_{0} t / \hbar}\right), \\
=-\frac{1}{\hbar^{2}} \int_{0}^{t_{2}} d \tau \operatorname{Tr}_{S \otimes R}\left(A \left[H_{I},\right.\right. & \\
& \left.\left.e^{-i H_{0} t / \hbar} B\left[\tilde{H}_{I}\left(\tau-t_{2}\right), \rho_{T}\left(t_{2}\right)\right] e^{i H_{0} t / \hbar}\right]\right),
\end{aligned}
$$

where the first order term in the interaction Hamiltonian coming from $\tilde{\rho}_{T}(0)$ term in Eq. (19) has been dropped because of Eq. (7). Since the product of two $H_{I}$ already appear explicitly in Eq. (20), we may then transform Eq. (20) into Heisenberg representation with the evolution equation $\exp (i H t / \hbar) \approx \exp \left(i H_{0} t / \hbar\right)+\mathcal{O}\left(H_{I}\right)$. Furthermore, by writing out the commutators explicitly and rearranging the Heisenberg operator terms, the resultant equation from Eq. (20) then becomes

$$
\begin{gathered}
-\frac{1}{\hbar^{2}} \int_{0}^{t_{2}} d \tau \operatorname{Tr}_{S \otimes R}\left(\left\{\tilde{H}_{I}\left(\tau-t_{1}\right)\left[H_{I}, A\right]\right\}\left(t_{1}\right) B\left(t_{2}\right) \rho_{T}(0)\right. \\
\left.+\left\{\left[A, H_{I}\right]\right\}\left(t_{1}\right)\left\{B \tilde{H}_{I}\left(\tau-t_{2}\right)\right\}\left(t_{2}\right) \rho_{T}(0)\right) .
\end{gathered}
$$

The first term in Eq. (21) is ready to combine with the second term in Eq. (17) to extend the integration from 0 to $t_{1}$. Similarly, one may rewrite the last term in Eq. (21) using the relation $B \tilde{H}_{I}\left(\tau-t_{2}\right)=\tilde{H}_{I}\left(\tau-t_{2}\right) B+\left[B, \tilde{H}_{I}(\tau-\right.$ $\left.t_{2}\right)$ ] so that the first new term can be combined with last term in Eq. (17) to extend the integration from 0 to $t_{1}$.

Putting all the resultant terms together, we obtain the evolution equation of the two-time CF's valid to second order in the interaction Hamiltonian as

$$
\begin{aligned}
& d\left\langle A\left(t_{1}\right) B\left(t_{2}\right)\right\rangle / d t_{1} \\
&=(i / \hbar) \operatorname{Tr}_{S \otimes R}\left(\left\{\left[H_{S}, A\right]\right\}\left(t_{1}\right) B\left(t_{2}\right) \rho_{T}(0)\right) \\
&+\frac{1}{\hbar^{2}} \int_{0}^{t_{1}} d \tau \operatorname{Tr}_{S \otimes R} \\
& \quad\left(\left\{\tilde{H}_{I}\left(\tau-t_{1}\right)\left[A, H_{I}\right]\right\}\left(t_{1}\right) B\left(t_{2}\right) \rho_{T}(0)\right. \\
&+ \\
&\left.+\left\{\left[H_{I}, A\right] \tilde{H}_{I}\left(\tau-t_{1}\right)\right\}\left(t_{1}\right) B\left(t_{2}\right) \rho_{T}(0)\right) \\
&+\frac{1}{\hbar^{2}} \int_{0}^{t_{2}} d \tau \operatorname{Tr}_{S \otimes R} \\
&\left(\left\{\left[H_{I}, A\right]\right\}\left(t_{1}\right)\left\{\left[B, \tilde{H}_{I}\left(\tau-t_{2}\right)\right]\right\}\left(t_{2}\right) \rho_{T}(0)\right) .
\end{aligned}
$$

Compared to Eq. (16), it is the existence of the last term in Eq. (22) that makes the QRT invalid. Equation (22) is the main result of this paper. The derivation is based on perturbative quantum master equation approach, so non-Markovian open quantum system models that are not exactly solvable can use our derived evolution equation to obtain the time evolutions of their two-time CF's of system operators, valid to second order in the systemenvironment interaction. In the derivation of Eqs. (16) and (22), we have also used the assumption of a factorized initial system-bath state $\rho_{T}(0)=\tilde{\rho}_{T}(0)=\rho(0) \otimes R_{0}$ and the condition of $\operatorname{Tr}_{R}\left[\tilde{H}_{I}(t) R_{0}\right]=0$, Eq. (7), to eliminate the first-order term. Since the form and nature of the Hamiltonians are not specified, Eq. (22) can be used to calculate the two-time CF's for non-Markovian open quantum systems with multi-level discrete or continuous Hilbert spaces, interacting with bosonic and/or fermionic environments. The procedure and the degrees of difficulty to apply Eq. (22) to a open quantum system model (by taking into account nonlocal bath memory effects and tracing out the bath degrees of freedom for factorized system-bath initial states) to obtain the two-time CF's of system operator are similar to those for the evaluation of the reduced density matrix of a secondorder time-convolutionless non-Markovian quantum master equation [e.g., Eq. (9)]. We will explicitly apply the evolution equation (22) to a general model of a quantum system coupled to a finite-temperature bosonic environment in Sec. III and a specific model of two-level system in Sec. IV Open quantum systems coupled to fermionic reservoirs (environments) could, for example, be quantum dots or other nanostructure systems coupled (connected) to nonequilibrium electron reservoirs (electrodes or leads) in the electron transport problems [31, 38 45]. The evolution equation (22) can also be used to calculate the non-Markovian two-time CF's in such systems. In summary, our evolution equation (22) can be applied 
to a wide range of system-environment models with any factorized (separable) system-environment initial states (pure or mixed).

\section{EVOLUTION EQUATIONS FOR THERMAL BOSONIC BATH MODELS}

To make contact with Refs. 18 20], we consider a quantum system coupled to a bosonic environment with a general Hamiltonian of the form

$$
H=H_{S}+\sum_{\lambda} \hbar g_{\lambda}\left(L^{\dagger} a_{\lambda}+L a_{\lambda}^{\dagger}\right)+\sum_{\lambda} \hbar \omega_{\lambda} a_{\lambda}^{\dagger} a_{\lambda}
$$

where the system coupling operator $L$ acts on the Hilbert space of the system, $a_{\lambda}$ and $a_{\lambda}^{\dagger}$ are the annihilation and creation operators on the bosonic environment Hilbert space, and $g_{\lambda}$ and $\omega_{\lambda}$ are respectively the coupling strength and the frequency of the $\lambda$ th environmental oscillator.

Applying Eq. (23) to Eqs. (16) and (22) and after tracing over the environmental degrees of freedom for factorized (separable) system-bath initial states, we arrive at the second-order evolution equations of the single-time expectation values

$$
\begin{aligned}
d & \left\langle A\left(t_{1}\right)\right\rangle / d t_{1} \\
=(i / \hbar) \operatorname{Tr}_{S}\left(\left\{\left[H_{S}, A\right]\right\}\left(t_{1}\right) \rho(0)\right) & t_{0}^{t_{1}} d \tau \operatorname{Tr}_{S} \\
+ & { }_{0} \alpha^{*}\left(t_{1}-\tau\right)\left\{\tilde{L}^{\dagger}\left(\tau-t_{1}\right)[A, L]\right\}\left(t_{1}\right) \rho(0) \\
& +\alpha\left(t_{1}-\tau\right)\left\{\left[L^{\dagger}, A\right] \tilde{L}\left(\tau-t_{1}\right)\right\}\left(t_{1}\right) \rho(0) \\
& +\beta^{*}\left(t_{1}-\tau\right)\left\{\tilde{L}\left(\tau-t_{1}\right)\left[A, L^{\dagger}\right]\right\}\left(t_{1}\right) \rho(0) \\
& \left.+\beta\left(t_{1}-\tau\right)\left\{[L, A] \tilde{L}^{\dagger}\left(\tau-t_{1}\right)\right\}\left(t_{1}\right) \rho(0)\right),
\end{aligned}
$$

and of the two-time CF's

$$
\begin{aligned}
d & \left\langle A\left(t_{1}\right) B\left(t_{2}\right)\right\rangle / d t_{1} \\
= & (i / \hbar) \operatorname{Tr}_{S}\left(\left\{\left[H_{S}, A\right]\right\}\left(t_{1}\right) B\left(t_{2}\right) \rho(0)\right) \\
+ & \int_{0}^{t_{1}} d \tau \operatorname{Tr}_{S} \\
& \left(\alpha^{*}\left(t_{1}-\tau\right)\left\{\tilde{L}^{\dagger}\left(\tau-t_{1}\right)[A, L]\right\}\left(t_{1}\right) B\left(t_{2}\right) \rho(0)\right. \\
& +\alpha\left(t_{1}-\tau\right)\left\{\left[L^{\dagger}, A\right] \tilde{L}\left(\tau-t_{1}\right)\right\}\left(t_{1}\right) B\left(t_{2}\right) \rho(0) \\
& +\beta^{*}\left(t_{1}-\tau\right)\left\{\tilde{L}\left(\tau-t_{1}\right)\left[A, L^{\dagger}\right]\right\}\left(t_{1}\right) B\left(t_{2}\right) \rho(0) \\
& \left.+\beta\left(t_{1}-\tau\right)\left\{[L, A] \tilde{L}^{\dagger}\left(\tau-t_{1}\right)\right\}\left(t_{1}\right) B\left(t_{2}\right) \rho(0)\right) \\
+ & \int_{0}^{t_{2}} d \tau \operatorname{Tr}_{S} \\
& \left(\alpha\left(t_{1}-\tau\right)\left\{\left[L^{\dagger}, A\right]\right\}\left(t_{1}\right)\left\{\left[B, \tilde{L}\left(\tau-t_{2}\right)\right]\right\}\left(t_{2}\right) \rho(0)\right. \\
+ & \left.\beta\left(t_{1}-\tau\right)\{[L, A]\}\left(t_{1}\right)\left\{\left[B, \tilde{L}^{\dagger}\left(\tau-t_{2}\right)\right]\right\}\left(t_{2}\right) \rho(0)\right)(25)
\end{aligned}
$$

Here $\tilde{L}(t)=\exp \left(i H_{S} t / \hbar\right) L \exp \left(-i H_{S} t / \hbar\right)$ is the system operator in the interaction picture with respect to $H_{S}$, and

$$
\begin{aligned}
& \alpha\left(t_{1}-\tau\right)=\sum_{\lambda}\left(\bar{n}_{\lambda}+1\right)\left|g_{\lambda}\right|^{2} e^{-i \omega_{\lambda}\left(t_{1}-\tau\right)}, \\
& \beta\left(t_{1}-\tau\right)=\sum_{\lambda} \bar{n}_{\lambda}\left|g_{\lambda}\right|^{2} e^{i \omega_{\lambda}\left(t_{1}-\tau\right)}
\end{aligned}
$$

are the environment CF's with $\alpha\left(t_{1}-\tau\right)=$ $\left\langle\sum_{\lambda} g_{\lambda} \tilde{a}_{\lambda}\left(t_{1}\right) \sum_{\lambda^{\prime}} g_{\lambda^{\prime}} \tilde{a}_{\lambda^{\prime}}^{\dagger}(\tau)\right\rangle_{R}$ and $\beta\left(t_{1}-\tau\right)=$ $\left\langle\sum_{\lambda} g_{\lambda} \tilde{a}_{\lambda}^{\dagger}\left(t_{1}\right) \sum_{\lambda^{\prime}} g_{\lambda^{\prime}} \tilde{a}_{\lambda^{\prime}}(\tau)\right\rangle_{R}$, where $\tilde{a}_{\lambda}\left(t_{1}\right)=$ $a_{\lambda} e^{-i \omega_{\lambda} t_{1}}$ and $\tilde{a}_{\lambda}^{\dagger}\left(t_{1}\right)=a_{\lambda}^{\dagger} e^{i \omega_{\lambda} t_{1}}$ are the environment operators in the interaction picture, and the symbol $\langle\cdots\rangle_{R}$ denotes taking a trace with respect to the density matrix of the thermal bosonic reservoir (environment). The thermal mean occupation number $\bar{n}_{\lambda}$ of the bosonic environment oscillators in Eqs. (26) and (27)) is $\bar{n}_{\lambda}=\left(e^{\hbar \omega_{\lambda} / k_{B} T}-1\right)^{-1}$.

The evolution equations (24) and (25) for a nonMarkovian bosonic environment have been presented in Ref. 22] without any derivation. In this paper, the detailed derivation of the evolution equations is given. Furthermore, the two-time CF evolution equation, Eq. (22), applicable for both bosonic and fermionic environments and applicable for more general form of systemenvironment interaction Hamiltonian has not been published in the literature yet.

As mentioned, the two-time evolution equations derived in Refs. [18 20] is, strictly speaking, applicable only for a zero-temperature environment. However, these equations were used to calculate the two-time CF's of system observables of dissipative spin-boson models at finite temperatures. This is possible only for the dissipative spin-boson models with Hermitian system coupling operators, $L=L^{\dagger}$. We will discuss this point in details in subsection IIIA In contrast, our bosonic evolution equations, Eqs. (24) and (25), are valid for both a Hermitian and a non-Hermitian system coupling operators and can be used to calculate the two-time CF's for any factorized (separable) system-reservoir initial state and for any arbitrary temperature as long as the assumption of the weak system-environment coupling still holds.

In Ref. 22], we used Eqs. (24) and (25) to calculate the finite-temperature single-time expectation values and two-time CF's for a non-Markovian pure-dephasing spinboson model of

$$
H_{S}=\left(\hbar \omega_{S} / 2\right) \sigma_{z}, \quad L=\sigma_{z}=L^{\dagger} .
$$

Since the non-Markovian dynamics of this exactly solvable pure-dephasing model can be cast into a time-local, convolutionless form and $\left[L, H_{S}\right]=0$, the results obtained by our second-order evolution equations turn out to be exactly the same as the exact results obtained by the direct operator evaluation. However, these results significantly differ from the non-Markovian twotime CF's obtained by wrongly directly applying the 
quantum regression theorem (QRT). This demonstrates the validity of the evolution equations (24) and (25). But the system coupling operators $L$ of this pure dephasing model [22] and the examples calculated in Refs. 18 20] are all Hermitian, i.e., $L^{\dagger}=L$. So we will present in Sec. IV the calculations of one-time averages and twotime CF's for a thermal spin-boson model with $L \neq L^{\dagger}$, for which only the evolution equations (24) and (25), rather than those in Refs. [18 20], are applicable.

\section{A. Comparison and discussion}

We discuss in the following the connection of our derived two-time evolution equation (25) with those presented in Refs. [18 20]. In Ref. [19], a master equation conditioned on initial coherent states of the environment in Bargmann representation, $\left(z_{0}, z_{0}^{\prime}\right)$, was derived in the weak system-environment coupling limit. Provided that the whole set of the initial conditions of the system of interest, $\left|\psi\left(z_{0}^{*}\right)\right\rangle$, and the statistical probability $\mathcal{J}\left(z_{0}, z_{0}^{*}\right)$ for the member $\left|\psi\left(z_{0}^{*}\right)\right\rangle\left|z_{0}\right\rangle$ of the statistical ensemble are known, this master equation with $z_{0}^{\prime}=z_{0}$ is capable of evaluating the evolution of single-time expectation values for general initial conditions, including initially correlated mixed states between the system and environment. However, the evolution equations of twotime (multi-time) CF's of system observables, Eq. (6) in Ref. [18], Eq. (31) in Ref. [19] and Eq. (60) in Ref. [20], were derived for an initial vacuum state of the environment and an initial pure state of the system of interest. As a result, these two-time evolution equations are valid only for a zero-temperature environment (if the system coupling operator is not Hermitian, i.e., $L \neq L^{\dagger}$; see discussions below). Compared with the corresponding zerotemperature two-time (multi-time) evolution equations derived in Refs. [18 20], our finite-temperature two-time evolution equation (25) is valid for any initial factorized (separable) states (pure or mixed) at finite temperatures and for both Hermitian and non-Hermitian system coupling operators. The extra terms containing the bath $\mathrm{CF}$ $\beta\left(t_{1}-\tau\right)$ or $\beta^{*}\left(t_{1}-\tau\right)$ are due to the finite-temperature thermal environment. If we take the zero-temperature limit by letting $\bar{n}_{\lambda}=0$ and thus $\beta\left(t_{1}-\tau\right)=\beta^{*}\left(t_{1}-\tau\right)=$ 0 , as well as consider the initial pure-state case by letting $\operatorname{Tr}_{S}[\cdots \rho(0)] \rightarrow\langle\psi(0)|\cdots| \psi(0)\rangle$, then Eqs.(24) and (25) reduce exactly to their corresponding zero-temperature pure-state evolution equations in Refs. [18 20].

However, calculations of the two-time CF's of system observables of dissipative spin-boson models in finite-temperature thermal baths (rather than zerotemperature baths) were presented in Refs. [18 20] even though in their derivations of the two-time (multi-time) evolution equations, the bath $\mathrm{CF}$ is given in its zerotemperature form,

$$
\alpha_{0}(t-\tau)=\sum_{\lambda}\left|g_{\lambda}\right|^{2} e^{-i \omega_{\lambda}\left(t_{1}-\tau\right)}
$$

This is only possible due to the reason that the system coupling operator is Hermitian, $L=L^{\dagger}$, in the thermal bath examples presented in Refs. [18 20]. One may understand this as follows. It is known that the finitetemperature density matrix operator of a thermal bath can be canonically mapped onto the zero-temperature density operator (the vacuum) of a larger (hypothetical) environment [46, 47]. By mapping the total Hamiltonian Eq. (23) and an initial thermal state to an extended total system with a vacuum state, the finite-temperature problem can be reduced to a zero-temperature problem, and the resultant pure state $\psi_{t}=\left|\psi_{t}\left(z^{*}, w^{*}\right)\right\rangle$ for the system of interest satisfies the following linear finite-temperature non-Markovian stochastic Schrödinger equation with two independent noises $z_{t}^{*}$ and $w_{t}^{*}$ [47, 48]:

$$
\begin{aligned}
\frac{\partial}{\partial t} \psi_{t}= & -i H_{S} \psi_{t}+L z_{t}^{*} \psi_{t}-L^{\dagger} \int_{0}^{t} d \tau \alpha(t-\tau) \frac{\delta \psi_{t}}{\delta z_{\tau}^{*}} \\
& +L^{\dagger} w_{t}^{*} \psi_{t}-L \int_{0}^{t} d \tau \beta(t-\tau) \frac{\delta \psi_{t}}{\delta w_{\tau}^{*}}
\end{aligned}
$$

where

$$
\begin{aligned}
& z_{t}^{*}=-i \sum_{\lambda} \sqrt{\bar{n}_{\lambda}+1} g_{\lambda}^{*} z_{\lambda}^{*} e^{i \omega_{\lambda} t}, \\
& w_{t}^{*}=-i \sum_{\lambda} \sqrt{\bar{n}_{\lambda}} g_{\lambda}^{*} w_{\lambda}^{*} e^{-i \omega_{\lambda} t}
\end{aligned}
$$

are two independent, colored, complex Gaussian noises with zero mean and satisfy

$$
\begin{aligned}
\mathcal{M}\left[z_{t} z_{\tau}\right]=\mathcal{M}\left[z_{t}^{*} z_{\tau}^{*}\right]=0, & \mathcal{M}\left[z_{t}^{*} z_{\tau}\right]=\alpha(t-\tau) ;(33) \\
\mathcal{M}\left[w_{t} w_{\tau}\right]=\mathcal{M}\left[w_{t}^{*} w_{\tau}^{*}\right]=0, & \mathcal{M}\left[w_{t}^{*} w_{\tau}\right]=\beta(t-\tau)(.34)
\end{aligned}
$$

Here, $z_{\lambda}^{*}$ and $w_{\lambda}^{*}$ are coherent state variables of the extended environment in Bargmann representation, $\mathcal{M}[\cdots]$ denotes the statistical average over the Gaussian processes $z_{t}^{*}$ and $w_{t}^{*}$, and the bath CF's $\alpha(t-\tau)$ and $\beta(t-\tau)$ are defined in Eqs. (26) and (27), respectively. In the zero-temperature limit $T \rightarrow 0$, the mean thermal occupation number of quanta in mode $\lambda$ approaches zero, i.e., $\bar{n}_{\lambda} \rightarrow 0$. and thus $\beta(t-\tau) \rightarrow 0$ and $\alpha(t-\tau) \rightarrow \alpha_{0}(t-\tau)$ that is defined in Eq. (29). In this case, the noises, Eqs. (31) and (32), become $z_{t}^{*}=-i \sum_{\lambda} g_{\lambda}^{*} z_{\lambda}^{*} e^{i \omega_{\lambda} t}$ and $w_{t}^{*}=0$, and the finite-temperature equation (30) reduces to the simple zero-temperature equation [47, 48]

$$
\frac{\partial}{\partial t} \psi_{t}=-i H_{S} \psi_{t}+L z_{t}^{*} \psi_{t}-L^{\dagger} \int_{0}^{t} d \tau \alpha_{0}(t-\tau) \frac{\delta \psi_{t}}{\delta z_{\tau}^{*}}
$$

Now consider the case of a Hermitian system coupling operator $L=L^{\dagger}$. The finite-temperature equation (30) can be simplified considerably by introducing the sum process $u_{t}^{*}=z_{t}^{*}+w_{t}^{*}$ that has a zero mean and satisfies

$$
\begin{aligned}
\mathcal{M}\left[u_{t} u_{\tau}\right] & =\mathcal{M}\left[u_{t}^{*} u_{\tau}^{*}\right]=0 \\
\mathcal{M}\left[u_{t}^{*} u_{\tau}\right] & =\alpha_{\mathrm{eff}}(t-\tau) \\
& =\alpha(t-\tau)+\beta(t-\tau) \\
& =\sum_{\lambda}\left|g_{\lambda}\right|^{2}\left\{\operatorname{coth}\left(\hbar \omega_{\lambda} / 2 k_{B} T\right) \cos \left[\omega_{\lambda}(t-\tau)\right]\right. \\
& \left.\quad-i \sin \left[\omega_{\lambda}(t-\tau)\right]\right\}
\end{aligned}
$$


In terms of the single noise process $u_{t}^{*}$, the linear finitetemperature non-Markovian stochastic Schrödinger equation (30) for the case of a Hermitian coupling operator $L=L^{\dagger}$ takes the simple form of the zero-temperature equation (35) with the replacement of $z_{t}^{*}$ by $u_{t}^{*}$ and $\alpha_{0}(t-\tau)$ by $\alpha_{\text {eff }}(t-\tau)=\alpha(t-\tau)+\beta(t-\tau)$ defined in Eq. (37). It is for this reason of the Hermitian coupling operator $L=L^{\dagger}=\sigma_{x}$ in the dissipative spin-boson model with a thermal environment that the two-time CF's of the system observables can be evaluated with the evolution equations derived in Refs. [18 20]. In other words, if the system operator coupled to the environment is not Hermitian $L \neq L^{\dagger}$, the two-time (multi-time) differential evolution equations presented in Refs. [18 20] are valid only for a zero-temperature environment.

In contrast, our two-time evolution equation (25) is valid for more general finite-temperature cases where the system coupling operator is not a Hermitian operator, i.e., $L \neq L^{\dagger}$. In the case of $L \neq L^{\dagger}$, our two-time evolution equation contains additional finitetemperature $\beta\left(t_{1}-\tau\right)$ and $\beta^{*}\left(t_{1}-\tau\right)$ terms which can not be combined and simplified to a simpler form as the zero-temperature evolution equation derived in Refs. 18 20]. For a Hermitian coupling operator $L=L^{\dagger}$, one can see that besides the replacement of a more general system state with an initial pure system state by letting $\langle\psi(0)|\cdots| \psi(0)\rangle \rightarrow \operatorname{Tr}_{S}[\cdots \rho(0)]$, the finite-temperature evolution equation (25) reduces to its zero-temperature counterpart in Refs. 18 20] with the effective bath CF given by $\alpha_{\text {eff }}=\alpha\left(t_{1}-\tau\right)+\beta\left(t_{1}-\tau\right)$ defined in Eq. (37). This demonstrates explicitly why the zero-temperature two-time evolution equations derived in Refs. 18 20] can be used to calculate the system operator CF's for a thermal spin-boson model with a Hermitian system coupling operator.

\section{B. Conditions for the QRT to hold}

As mentioned in subsection IB, QRT is a very useful procedure that enables one (in certain circumstances) to calculate for two-time (multi-time) CF's of system operators from the knowledge of the evolution equations of single-time expectation values. One can notice that if the last two terms in Eq. (25) vanish, then the non-Markovian single-time and two-time evolution equations (24) and (25) will have the same form with the same evolution coefficients and thus the QRT can apply. As expected, these two terms vanish in the Markovian case since the time integration of the corresponding $\delta$-correlated reservoir CF's, $\alpha\left(t_{1}-\tau\right) \propto \delta\left(t_{1}-\tau\right)$ and $\beta\left(t_{1}-\tau\right) \propto \delta\left(t_{1}-\tau\right)$, over the variable $\tau$ in the domain from 0 to $t_{2}$ is zero as $t_{1}>t_{2}$. So from Eq. (25), in the weak system-environment coupling case the QRT holds when (i) $\left[L^{\dagger}, A\right]=0$ or $\left[B, \tilde{L}\left(\tau-t_{2}\right)\right]=0$ at the zero temperature, (ii) at finite temperatures, in addition to condition (i), the following condition also needs to be satisfied: $[L, A]=0$ or $\left[B, \tilde{L}^{\dagger}\left(\tau-t_{2}\right)\right]=0$, (iii) in the Markovian case where the bath CF's are $\delta$-correlated in time.

Note that in some models, certain CF's, which formally obey the QRT but with evolution equations coupled with those of other CF's that do not obey the QRT, may yield solutions different from those given by the QRT [19].

\section{APPLICATION TO A THERMAL SPIN BOSON MODEL WITH $L \neq L^{\dagger}$}

To illustrate the usage of the equations we have derived, we apply them to the problem of a two-level system coupled to a thermal reservoir, in which $L \neq L^{\dagger}$. We consider Hamiltonian, Eq. (23), with $H_{S}=\left(\hbar \omega_{A} / 2\right) \sigma_{z}$, a coupling operator $L=\sigma_{-}$and a system-environment interaction Hamiltonian whose magnitude is small enough to be considered as a perturbation. Since the coupling operator $L \neq L^{\dagger}$ is not Hermitian, the two-time (multitime) evolution equations derived in Refs. [18 20] are not applicable and our evolution equation (25) should be employed to obtain the time evolutions of the two-time CF's.

\section{A. Single-time expectation values}

Before calculating the two-time CF's, it is instructive to obtain the master equation of the reduced system density matrix for the model. Transferring from the interaction picture back to the Schrödinger picture for Eq. (9) and using the general Hamiltonian, Eq. (23), we obtain the second-order time-convolutionless nonMarkovian master equation for the reduced density matrix $\rho(t)$ as

$$
\begin{aligned}
& \frac{d \rho(t)}{d t}=\frac{1}{i \hbar}\left[H_{S}, \rho(t)\right] \\
& -\int_{0}^{t} d \tau\left\{\alpha(t-\tau)\left[L^{\dagger} \tilde{L}(\tau-t) \rho(t)-\tilde{L}(\tau-t) \rho(t) L^{\dagger}\right]\right. \\
& \quad+\beta(t-\tau)\left[L \tilde{L}^{\dagger}(\tau-t) \rho(t)-\tilde{L}^{\dagger}(\tau-t) \rho(t) L\right] \\
& \quad+\text { h.c. }\}
\end{aligned}
$$

where $\alpha(t-\tau)$ and $\beta(t-\tau)$ are defined in Eqs. (26) and (27) respectively, h.c. indicates the hermitian conjugate of previous terms, and an operator with a tilde on the top indicates that it is an operator in the interaction picture. The only real assumption used to obtain Eq. (38) valid to second order in the system-environment coupling strength is the total density matrix factorizing at the initial time $t=0, \rho_{T}(0)=\rho(0) \otimes R_{0}$. Taking $L=\sigma_{-}$, $L^{\dagger}=\sigma_{+}$, then $\tilde{L}(t)=\sigma_{-} e^{-i \omega_{A} t}$, and $\tilde{L}^{\dagger}(t)=\sigma_{+} e^{i \omega_{A} t}$, and substituting them into Eq. (38), we obtain

$$
\begin{aligned}
& \frac{d \rho(t)}{d t}=-i \frac{\omega_{A}}{2}\left[\sigma_{z}, \rho(t)\right] \\
& \quad-\left\{\Gamma_{1}(t)\left(\sigma_{+} \sigma_{-} \rho(t)-\sigma_{-} \rho(t) \sigma_{+}\right)\right. \\
& \left.\quad+\Gamma_{2}(t)\left(\sigma_{-} \sigma_{+} \rho(t)-\sigma_{+} \rho(t) \sigma_{-}\right)+\text {h.c. }\right\}
\end{aligned}
$$


where

$$
\begin{aligned}
& \Gamma_{1}(t)=\int_{0}^{t} d \tau \alpha(t-\tau) e^{+i \omega_{A}(t-\tau)}, \\
& \Gamma_{2}(t)=\int_{0}^{t} d \tau \beta(t-\tau) e^{-i \omega_{A}(t-\tau)} .
\end{aligned}
$$

The master equation (39) is a time-local and convolutionless differential equation. The effect of the non-Markovian environment on the second-order master equation (39) is taken into account by the timedependent coefficients $\Gamma_{1}\left(t_{1}\right)$ and $\Gamma_{2}\left(t_{1}\right)$ defined in Eqs. (40) and (41) instead of memory integrals. Likewise, the evolution equations of single-time expectation values and two-time CF's of system operators valid to the second order are also expected to be convolutionless. Taking again $L=\sigma_{-}, L^{\dagger}=\sigma_{+}$, then $\tilde{L}(t)=\sigma_{-} e^{-i \omega_{A} t}$, and $\tilde{L}^{\dagger}(t)=\sigma_{+} e^{i \omega_{A} t}$, and using the commutation relation between the Pauli matrices, we obtain straightforwardly from Eq. (24) the following evolution equations of the single-time expectation values of system operators as

$$
\begin{aligned}
d\left\langle\sigma_{+}\left(t_{1}\right)\right\rangle / d t_{1}= & i \omega_{A}\left\langle\sigma_{+}\left(t_{1}\right)\right\rangle \\
& -\left[\Gamma_{1}^{*}\left(t_{1}\right)+\Gamma_{2}\left(t_{1}\right)\right]\left\langle\sigma_{+}\left(t_{1}\right)\right\rangle \\
d\left\langle\sigma_{-}\left(t_{1}\right)\right\rangle / d t_{1}= & -i \omega_{A}\left\langle\sigma_{-}\left(t_{1}\right)\right\rangle \\
& -\left[\Gamma_{1}\left(t_{1}\right)+\Gamma_{2}^{*}\left(t_{1}\right)\right]\left\langle\sigma_{-}\left(t_{1}\right)\right\rangle \\
d\left\langle\sigma_{z}\left(t_{1}\right)\right\rangle / d t_{1}=- & {\left[\Gamma_{1}\left(t_{1}\right)+\Gamma_{1}^{*}\left(t_{1}\right)+\Gamma_{2}^{*}\left(t_{1}\right)+\Gamma_{2}\left(t_{1}\right)\right] } \\
& \times\left\langle\sigma_{z}\left(t_{1}\right)\right\rangle \\
& -\left[\Gamma_{1}\left(t_{1}\right)+\Gamma_{1}^{*}\left(t_{1}\right)-\Gamma_{2}^{*}\left(t_{1}\right)-\Gamma_{2}\left(t_{1}\right)\right] .
\end{aligned}
$$

Equations (42)-(44) can also be obtained directly from the master equation (39) through $d\left\langle A\left(t_{1}\right)\right\rangle / d t_{1}=$ $\operatorname{Tr}_{S}\left[A\left(d \rho\left(t_{1}\right) / d t_{1}\right)\right]$.

\section{B. Two-time CF's}

For the evaluations of the two-time CF's of the system observables, we consider the following four cases.

Case 1: $\left[L^{\dagger}, A\right]=0$ or $[B, \tilde{L}(t)]=0$; and $[L, A]=0$ or $\left[B, \tilde{L}^{\dagger}(t)\right]=0$. In this case, let $A=\sigma_{i}, B=$ $\sigma_{i}$ with $i=+,-$. Applying the commutation relations between the Pauli matrices and the definition of $\operatorname{Tr}_{S}\left[\sigma_{i}\left(t_{1}\right) \sigma_{i}\left(t_{2}\right) \rho(0)\right]=\left\langle\sigma_{i}\left(t_{1}\right) \sigma_{i}\left(t_{2}\right)\right\rangle$ to the right-hand side of the two-time evolution equation (25), we then obtain

$$
\begin{aligned}
d\left\langle\sigma_{+}\left(t_{1}\right) \sigma_{+}\left(t_{2}\right)\right\rangle / d t_{1}= & i \omega_{A}\left\langle\sigma_{+}\left(t_{1}\right) \sigma_{+}\left(t_{2}\right)\right\rangle \\
& -\left[\Gamma_{1}^{*}\left(t_{1}\right)+\Gamma_{2}\left(t_{1}\right)\right]\left\langle\sigma_{+}\left(t_{1}\right) \sigma_{+}\left(t_{2}\right)\right\rangle,
\end{aligned}
$$$$
\begin{aligned}
d\left\langle\sigma_{-}\left(t_{1}\right) \sigma_{-}\left(t_{2}\right)\right\rangle / d t_{1}= & -i \omega_{A}\left\langle\sigma_{-}\left(t_{1}\right) \sigma_{-}\left(t_{2}\right)\right\rangle \\
& -\left[\Gamma_{1}\left(t_{1}\right)+\Gamma_{2}^{*}\left(t_{1}\right)\right]\left\langle\sigma_{-}\left(t_{1}\right) \sigma_{-}\left(t_{2}\right)\right\rangle,
\end{aligned}
$$

where $\Gamma_{1}\left(t_{1}\right)$ and $\Gamma_{2}\left(t_{1}\right)$ are defined in Eqs. (40) and (41), respectively. One can see that the evolution equations of the two-time CF's, Eqs. (45) and (46), have the same forms as the evolution equations of the single-time expectation values $\left\langle\sigma_{+}\left(t_{1}\right)\right\rangle$ and $\left\langle\sigma_{-}\left(t_{1}\right)\right\rangle$, Eqs. (42) and (43), respectively. Hence the QRT holds in this case.

Case 2: $[A, L]=0$ or $[B, \tilde{L}(t)]=0$. In this case, using Eq. (25), we obtain

$$
\begin{aligned}
d\left\langle\sigma_{-}\left(t_{1}\right) \sigma_{z}\left(t_{2}\right)\right\rangle / d t_{1}= & -i \omega_{A}\left\langle\sigma_{-}\left(t_{1}\right) \sigma_{z}\left(t_{2}\right)\right\rangle \\
& -\left[\Gamma_{1}\left(t_{1}\right)+\Gamma_{2}^{*}\left(t_{1}\right)\right]\left\langle\sigma_{-}\left(t_{1}\right) \sigma_{z}\left(t_{2}\right)\right\rangle \\
& -2 \Gamma_{3}\left(t_{1}, t_{2}\right)\left\langle\sigma_{z}\left(t_{1}\right) \sigma_{-}\left(t_{2}\right)\right\rangle, \quad(47) \\
d\left\langle\sigma_{z}\left(t_{1}\right) \sigma_{-}\left(t_{2}\right)\right\rangle / d t_{1}= & -\left[\Gamma_{1}\left(t_{1}\right)+\Gamma_{1}^{*}\left(t_{1}\right)+\Gamma_{2}^{*}\left(t_{1}\right)+\Gamma_{2}\left(t_{1}\right)\right] \\
& \times\left\langle\sigma_{z}\left(t_{1}\right) \sigma_{-}\left(t_{2}\right)\right\rangle \\
& -\left[\Gamma_{1}\left(t_{1}\right)+\Gamma_{1}^{*}\left(t_{1}\right)-\Gamma_{2}^{*}\left(t_{1}\right)-\Gamma_{2}\left(t_{1}\right)\right] \\
& \times\left\langle\sigma_{-}\left(t_{2}\right)\right\rangle \\
& -2 \Gamma_{4}\left(t_{1}, t_{2}\right)\left\langle\sigma_{-}\left(t_{1}\right) \sigma_{z}\left(t_{2}\right)\right\rangle,
\end{aligned}
$$

where

$$
\begin{aligned}
& \Gamma_{3}\left(t_{1}, t_{2}\right)=\int_{0}^{t_{2}} d \tau \alpha\left(t_{1}-\tau\right) e^{i \omega_{A}\left(t_{2}-\tau\right)} \\
& \Gamma_{4}\left(t_{1}, t_{2}\right)=\int_{0}^{t_{2}} d \tau \beta\left(t_{1}-\tau\right) e^{-i \omega_{A}\left(t_{2}-\tau\right)}
\end{aligned}
$$

When we obtain Eq. (47) from Eq. (25), the last term containing $\Gamma_{3}\left(t_{1}, t_{2}\right)$ in Eq. (47) does not vanish since $\left[L^{\dagger}, A\right] \neq 0$ and $\left[B, \tilde{L}\left(\tau-t_{2}\right)\right] \neq 0$. Similarly, when we obtain Eq. (48) from Eq. (25), because $[L, A] \neq 0$ and $\left[B, \tilde{L}^{\dagger}\left(\tau-t_{2}\right)\right] \neq 0$, the last term containing $\Gamma_{4}\left(t_{1}, t_{2}\right)$ in Eq. (48) exists. Thus, compared with single-time equations (43) and (44), the two-time equations (47) and (48) have the extra last terms containing $\Gamma_{3}\left(t_{1}, t_{2}\right)$ and $\Gamma_{4}\left(t_{1}, t_{2}\right)$, respectively. As a result, the QRT does not hold in this case. It is also obvious from the sole appearance of the individual coefficient of either $\Gamma_{3}\left(t_{1}, t_{2}\right)$ in Eq. (47) or $\Gamma_{4}\left(t_{1}, t_{2}\right)$ in Eq. (48) that the finitetemperature bath CF's $\alpha\left(t_{1}-\tau\right)$ and $\beta\left(t_{1}-\tau\right)$ can not be combined into the single effective bath CF $\alpha_{\text {eff }}\left(t_{1}-\tau\right)=$ $\alpha\left(t_{1}-\tau\right)+\beta\left(t_{1}-\tau\right)$ of Eq. (37) as in the Hermitian coupling operator case.

Case 3: $\left[A, L^{\dagger}\right]=0$ or $\left[B, \tilde{L}^{\dagger}(t)\right]=0$. Using Eq. (25), we obtain for this case

$$
\begin{aligned}
d\left\langle\sigma_{+}\left(t_{1}\right) \sigma_{z}\left(t_{2}\right)\right\rangle / d t_{1}= & +i \omega_{A}\left\langle\sigma_{+}\left(t_{1}\right) \sigma_{z}\left(t_{2}\right)\right\rangle \\
& -\left[\Gamma_{1}^{*}\left(t_{1}\right)+\Gamma_{2}\left(t_{1}\right)\right]\left\langle\sigma_{+}\left(t_{1}\right) \sigma_{z}\left(t_{2}\right)\right\rangle \\
& -2 \Gamma_{4}\left(t_{1}, t_{2}\right)\left\langle\sigma_{z}\left(t_{1}\right) \sigma_{+}\left(t_{2}\right)\right\rangle, \quad(51) \\
d\left\langle\sigma_{z}\left(t_{1}\right) \sigma_{+}\left(t_{2}\right)\right\rangle / d t_{1}=- & {\left[\Gamma_{1}\left(t_{1}\right)+\Gamma_{1}^{*}\left(t_{1}\right)+\Gamma_{2}^{*}\left(t_{1}\right)+\Gamma_{2}\left(t_{1}\right)\right] } \\
& \times\left\langle\sigma_{z}\left(t_{1}\right) \sigma_{+}\left(t_{2}\right)\right\rangle \\
& -\left[\Gamma_{1}\left(t_{1}\right)+\Gamma_{1}^{*}\left(t_{1}\right)-\Gamma_{2}^{*}\left(t_{1}\right)-\Gamma_{2}\left(t_{1}\right)\right] \\
& \times\left\langle\sigma_{+}\left(t_{2}\right)\right\rangle \\
& -2 \Gamma_{3}\left(t_{1}, t_{2}\right)\left\langle\sigma_{+}\left(t_{1}\right) \sigma_{z}\left(t_{2}\right)\right\rangle .
\end{aligned}
$$

Similarly, compared with the single-time evolution equations (42) and (44), the two-time evolution equations (51) and (52) have the extra last terms containing $\Gamma_{4}^{*}\left(t_{1}\right)$ and $\Gamma_{3}\left(t_{1}\right)$, respectively. As a result, the QRT also does not hold for these two CF's. 
Case 4: $[A, L] \neq 0,\left[A, L^{\dagger}\right] \neq 0$ and $[B, \tilde{L}(t)] \neq 0$, $\left[B, \tilde{L}^{\dagger}(t)\right] \neq 0$. In this case, by using Eq. (25), we obtain the following equations

$$
\begin{aligned}
d\left\langle\sigma_{z}\left(t_{1}\right) \sigma_{z}\left(t_{2}\right)\right\rangle / d t_{1}=- & {\left[\Gamma_{1}\left(t_{1}\right)+\Gamma_{1}^{\dagger}\left(t_{1}\right)+\Gamma_{2}^{\dagger}\left(t_{1}\right)+\Gamma_{2}\left(t_{1}\right)\right] } \\
& \times\left\langle\sigma_{z}\left(t_{1}\right) \sigma_{z}\left(t_{2}\right)\right\rangle \\
- & {\left[\Gamma_{1}\left(t_{1}\right)+\Gamma_{1}^{\dagger}\left(t_{1}\right)-\Gamma_{2}^{\dagger}\left(t_{1}\right)-\Gamma_{2}\left(t_{1}\right)\right] } \\
& \times\left\langle\sigma_{z}\left(t_{2}\right)\right\rangle \\
& +4 \Gamma_{3}\left(t_{1}, t_{2}\right)\left\langle\sigma_{+}\left(t_{1}\right) \sigma_{-}\left(t_{2}\right)\right\rangle \\
& +4 \Gamma_{4}\left(t_{1}, t_{2}\right)\left\langle\sigma_{-}\left(t_{1}\right) \sigma_{+}\left(t_{2}\right)\right\rangle .
\end{aligned}
$$

The evolution equation of the CF $\left\langle\sigma_{z}\left(t_{1}\right) \sigma_{z}\left(t_{2}\right)\right\rangle$, Eq. (53), is coupled with the evolution equations of the CF's $\left\langle\sigma_{+}\left(t_{1}\right) \sigma_{-}\left(t_{2}\right)\right\rangle$ and $\left\langle\sigma_{-}\left(t_{1}\right) \sigma_{+}\left(t_{2}\right)\right\rangle$, which correspond to the CF's in Case 2 and Case 3, respectively. Their evolution equations, obtained from Eq. (25), are

$$
\begin{aligned}
d\left\langle\sigma_{-}\left(t_{1}\right) \sigma_{+}\left(t_{2}\right)\right\rangle / d t_{1}= & -i \omega_{A}\left\langle\sigma_{-}\left(t_{1}\right) \sigma_{+}\left(t_{2}\right)\right\rangle \\
& -\left[\Gamma_{1}\left(t_{1}\right)+\Gamma_{2}^{*}\left(t_{1}\right)\right]\left\langle\sigma_{-}\left(t_{1}\right) \sigma_{+}\left(t_{2}\right)\right\rangle \\
& +\Gamma_{3}\left(t_{1}, t_{2}\right)\left\langle\sigma_{z}\left(t_{1}\right) \sigma_{z}\left(t_{2}\right)\right\rangle, \quad(54) \\
d\left\langle\sigma_{+}\left(t_{1}\right) \sigma_{-}\left(t_{2}\right)\right\rangle / d t_{1}= & i \omega_{A}\left\langle\sigma_{+}\left(t_{1}\right) \sigma_{-}\left(t_{2}\right)\right\rangle \\
& -\left[\Gamma_{1}^{*}\left(t_{1}\right)+\Gamma_{2}\left(t_{1}\right)\right]\left\langle\sigma_{+}\left(t_{1}\right) \sigma_{-}\left(t_{2}\right)\right\rangle \\
& +\Gamma_{4}\left(t_{1}, t_{2}\right)\left\langle\sigma_{z}\left(t_{1}\right) \sigma_{z}\left(t_{2}\right)\right\rangle .
\end{aligned}
$$

From Eqs. (53), (54) and (55), it is obvious that the QRT also does not hold for CF's $\left\langle\sigma_{z}\left(t_{1}\right) \sigma_{z}\left(t_{2}\right)\right\rangle,\left\langle\sigma_{+}\left(t_{1}\right) \sigma_{-}\left(t_{2}\right)\right\rangle$ and $\left\langle\sigma_{-}\left(t_{1}\right) \sigma_{+}\left(t_{2}\right)\right\rangle$.

We may consider any spectral density for which the time-convolutionless perturbation theory is still valid to characterize the environment, but for simplicity we consider a spectral density $J(\omega)=\sum_{\lambda}\left|g_{\lambda}\right|^{2} \delta\left(\omega-\omega_{\lambda}\right)=$ $\gamma \hbar \omega(\omega / \Lambda)^{n-1} \exp \left(-\omega^{2} / \Lambda^{2}\right)$ with $n=1$ (Ohmic), where $\Lambda$ is the cut-off frequency and $\gamma$ is a dimensionless constant characterizing the interaction strength to the environment. Figure 1 shows the real part of the time evolution of the system operator CF's $\left\langle\sigma_{+}\left(t_{1}\right) \sigma_{-}\left(t_{2}\right)\right\rangle$ and $\left\langle\sigma_{z}\left(t_{1}\right) \sigma_{z}\left(t_{2}\right)\right\rangle$, as well as the Fourier spectrum $S(\omega)$ of $\left\langle\sigma_{+}\left(t_{1}\right) \sigma_{-}\left(t_{2}\right)\right\rangle$. The CF's are obtained in three different cases: the first is in the Markovian case [i.e, taking the reservoir CF's $\alpha\left(t_{1}-\tau\right)$ and $\beta\left(t_{1}-\tau\right)$ in Eq. (25) to be $\delta$-correlated in time, or equivalently taking the coefficients of $\Gamma_{1}, \Gamma_{1}^{\dagger}, \Gamma_{2}$, and $\Gamma_{2}^{\dagger}$ to be time-independent and equal to their Markovian long-time values and setting all $\Gamma_{3}\left(t_{1}, t_{2}\right)$ and $\Gamma_{4}\left(t_{1}, t_{2}\right)$ to be zero in Eqs. (53), (54) and (55)], the second is in the non-Markovian case with a finite cut-off frequency but wrongly directly using the QRT method [i.e., the last two terms of Eq. (25) or equivalently the terms containing $\Gamma_{3}\left(t_{1}, t_{2}\right)$ or $\Gamma_{4}\left(t_{1}, t_{2}\right)$ in Eqs. (53), (54) and (55) being all neglected], and the third is in the non-Markovian case with a finite cutoff frequency [i.e., using the evolution equation (25) or equivalently Eqs. (53), (54) and (55) derived in this paper]. The initial environmental state is in the thermal state and the system state in Fig. 1(a)-(c) is arbitrarily chosen to be $|\Psi\rangle=\left(\frac{\sqrt{3}}{2}|e\rangle+\frac{1}{2}|g\rangle\right)$. We can see
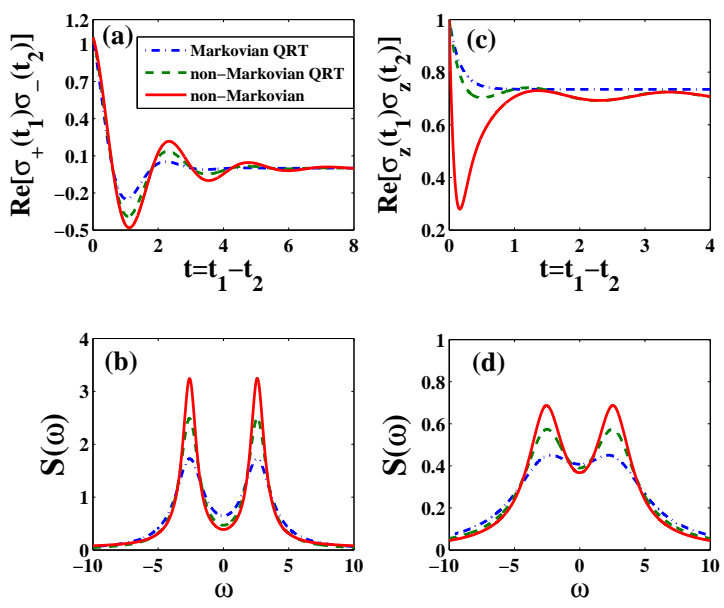

FIG. 1. (Color online) (a) Real part of the time evolution and (b) Fourier spectrum $S(\omega)$ of the system operator $\mathrm{CF}$ $\left\langle\sigma_{+}\left(t_{1}\right) \sigma_{-}\left(t_{2}\right)\right\rangle$, and (c) real part of $\left\langle\sigma_{z}\left(t_{1}\right) \sigma_{z}\left(t_{2}\right)\right\rangle$ for three different cases: Markovian using the QRT (blue dot-dashed line), non-Markovian using the QRT (green dashed line) and non-Markovian (red solid line) using the evolution equation (25). Other parameters used are $\omega_{A}=3,\left(k_{B} T / \hbar\right)=1$, $\Lambda=5, \gamma=0.1$, and $t_{2}=1$. (d) Fourier spectrum $S(\omega)$ of $\left\langle\sigma_{+}\left(t_{1}\right) \sigma_{-}\left(t_{2}\right)\right\rangle$ for a different parameter of $\gamma=0.35$ and for an initial mixed system state. The results of the nonMarkovian QRT case and the non-Markovian evolution case in (c) become indistinguishable when $t=t_{1}-t_{2}$ is larger than 1.5 .

that there are considerable differences between the results obtained in the three different cases in Fig. 1(a) and (b), and more significant differences can be observed in Fig. 1(c) and (d). The oscillations of the CF's are more pronounced in the non-Markovian cases. In Fig. 1(b), the coherent peaks of the Fourier spectrum centered at $\omega= \pm \omega_{A}$ are higher and the widths are narrower in the non-Markovian cases. The CF $\left\langle\sigma_{z}\left(t_{1}\right) \sigma_{z}\left(t_{2}\right)\right\rangle$ of the nonMarkovian evolution equation case (in red solid line) in Fig. 1(c) differs more from the CF's of the other two cases (in green dashed line and in blue dot-dashed line) in the short-time regime than the CF $\left\langle\sigma_{+}\left(t_{1}\right) \sigma_{-}\left(t_{2}\right)\right\rangle$ of the non-Markovian evolution equation case (in red solid line) in Fig. 1(a) does. This is because as compared to the evolution equation of $\left\langle\sigma_{+}\left(t_{1}\right) \sigma_{-}\left(t_{2}\right)\right\rangle$ of Eq. (55), the evolution equation of $\left\langle\sigma_{z}\left(t_{1}\right) \sigma_{z}\left(t_{2}\right)\right\rangle$ of Eq. (53) has, in addition to a term proportional to $\Gamma_{4}\left(t_{1}, t_{2}\right)$, an extra correction term proportional to $\Gamma_{3}\left(t_{1}, t_{2}\right)$ over its QRT counterparts. It is also found that generally the results of the non-Markovian QRT case (in green dashed lines) approach those of the non-Markovian evolution equation case (in red solid lines) more closely than the results of the Markovian QRT case (in blue dot-dashed lines) do. Similar behaviors are also observed when the temperature is increased or when the cut-off frequency $\Lambda$ is increased. The Markovian case can be recovered from the 
non-Markovian ones in the limit when the cut-off frequency $\Lambda \rightarrow \infty$, in which the three results coincide. For a larger $\gamma$ and for an initial mixed system state with the values of the off-diagonal density matrix elements being a quarter of those of the pure state $|\Psi\rangle$, the peak heights of $S(\omega)$ are lower as shown in Fig. 1(d). Furthermore in Fig. 11(d), the two coherent peaks are still clearly visible in the non-Markovian cases, while the two peaks is barely visible in the Markovian case.

For the present spin-boson model with the system coupling operator $L \neq L^{\dagger}$, the self-Hamiltonian of the spin does not commute with the system coupling operator, i.e, $\left[H_{S}, L\right] \neq 0$, and the environment coupling operator also does not commute with the self-Hamiltonian of the environment, i.e., $\left[H_{R}, a_{\lambda}\right] \neq 0$. Thus the exact non-Markovian finite-temperature two-time CF's of the present spin-boson model are not directly available. But in Ref. 22], we evaluated the exact non-Markovian finitetemperature two-time CF's of the system operators for an exactly solvable pure-dephasing spin-boson model in two ways, one by exact direct operator technique without any approximation and the other by the derived evolution equation (25) valid to second order in the systemenvironment interaction Hamiltonian. The perfect agreement of the results between the non-Markovian evolution equation case and the exact operator evaluation case, and the significant difference between the non-Markovian evolution equation case and the case of wrongly applying non-Markovian QRT [22]. demonstrate clearly the validity of the derived evolution equation (25). It is thus believed that in the weak system-environment coupling limit, the finite-temperature CF's calculated using our evolution equation that takes into account the nonlocal environment memory term, Eq. (18), for the present spinboson model would agree more closely with the exact results than those in the non-Markoian QRT and Markovian QRT cases.

\section{CONCLUSION}

In summary, we have derived evolution equations of the single-time and two-time CF's of system operators, using a quantum master equation technique different from those presented in Refs. [18 20]. This quantum master equation approach allows us to explicitly point out an important nonlocal environment (bath) memory term that vanishes in the Markovian case but makes the evolution equation deviate from the QRT in general cases. The derived two-time equations are valid for thermal environments at any temperature with Hermitian or nonHermitian coupling operators and for any initially factorized (separable) system-reservoir state (pure or mixed) as long as the assumption of Eq. (7) and the approximation of the weak system-environment coupling that are used to derive the equations apply. In contrast to the evolution equations presented in Refs. [18 20, 22] that are applicable for bosonic environments, Eq. (22) derived in this paper can be used to calculate the two-time CF's for a wide range of system-environment models with bosonic and/or fermionic environments. We have also given conditions on which the QRT holds in the weak system-environment coupling case and have applied the derived equations to a problem of a two-level system (atom) coupled to a finitetemperature thermal bosonic environment (electromagnetic fields), in which the system coupling operator is not Hermitian, $L \neq L^{\dagger}$, and the evolution equations derived in Refs. [18 20 are not applicable. It is easy to calculate the two-time CF's using the derived evolution equations. Other non-Markovian open quantum system models that are not exactly solvable can be proceeded in a similar way to obtain the time evolutions of their two-time system operator CF's valid to second order in the system-environment interaction Hamiltonian. This illustrates the practical usage of the evolution equations. Therefore, the derived evolution equations that generalize the QRT to the non-Markovian cases will have broad applications in many different branches of physics, such as quantum optics, statistical physics, chemical physics, quantum transport in nanostructure devices and so forth when the properties related to the two-time CF's are of interests.

\section{ACKNOWLEDGMENTS}

We would like to acknowledge support from the National Science Council, Taiwan, under Grant No. 972112-M-002-012-MY3, support from the Frontier and Innovative Research Program of the National Taiwan University under Grants No. 99R80869 and No. 99R80871, and support from the focus group program of the $\mathrm{Na}$ tional Center for Theoretical Sciences, Taiwan. H.S.G. is grateful to the National Center for High-performance Computing, Taiwan, for computer time and facilities.
[1] M. O. Scully and M. S. Zubairy, Quantum Optics, (Cambridge, 1997).

[2] H. J. Carmichael, Statistical Methods in Quantum Optics 1 (Springer, Berlin, 1999).

[3] S. T. Barnett and P. M. Radmore, Methods in theoretical quantum optics (Claredon Press, Oxford, 2002).
[4] C. W. Gardiner and P. Zoller, Quantum Noise, 2nd edition. (Springer-Verlag, Berlin, 2000).

[5] D. F. Walls and G. J. Milburn, Quantum Optics, 2nd edition. (Springer-Verlag, Berlin, 2008).

[6] J. P. Paz and W. H. Zurek in Coherent Matter Waves, Proceedings of the Les Houches Summer School, Session LXXII, edited by R. Kaiser, C. Westbrook, 
and F. David (Springer-Verlag, Berlin, 2001 ); arXiv: quant-ph/0010011

[7] H.P. Breuer and F. Petruccione, The Theory of Open Quantum Systems (Oxford University Press, Oxford, 2002).

[8] H. Grabert, P. Schramm and G.-L. Ingold, Phys. Rep. 168, 115 (1988).

[9] U. Weiss, Quantum Dissipative Systems, 3rd edition. (World Scientific, Singapore, 2008).

[10] Y. M. Blanter and M. Büttiker, Phys. Rep. 336, 1 (2000).

[11] A. A. Clerk, M. H. Devoret, S. M. Girvin, F. Marquardt, and R. J. Schoelkopf, Rev. Mod. Phys. 82, 1155 (2010)

[12] H. Grabert, Z. Phys. B 49, 161 (1982).

[13] P. Talkner, Ann. Phys. (N.Y.) 167, 390 (1995).

[14] G. W. Ford and R. F. O'Connell, Phys. Rev. Lett. 77, 798 (1996).

[15] G. W. Ford and R. F. O'Connell, Ann. Phys. (N.Y.) 276, 144 (1999); G. W. Ford and R. F. O'Connell, Opt. Commun. 179, 451 (2000).

[16] M. Lax, Opt. Commun. 179, 463 (2000).

[17] G. W. Ford and R. F. O'Connell, Opt. Commun. 179, 477 (2000).

[18] D. Alonso and I. de Vega, Phys. Rev. Lett. 94, 200403(2005).

[19] I. de Vega and D. Alonso, Phys. Rev. A 73, 022102(2006).

[20] D. Alonso and I. de Vega, Phys. Rev.A 75, 052108(2007).

[21] I. de Vega and D. Alonso, Phys. Rev. A 77, 043836 (2008).

[22] H.-S. Goan, C.-C. Jian, and P.-W. Chen, Phys. Rev. A 82, 012111 (2010).

[23] In Ref. [22], the subscript of $S \oplus R$ attached to the trace symbol of Tr was used to represent a trace over the degrees of freedom of the total composite system. Here, a more commonly used notation of $S \otimes R$ is adopted for a trace over the direct-tensor-product Hilbert space of the system and the reservoir.

[24] C. Meier and D. J. Tannor, J. Chem. Phys. 111, 3365 (1999).

[25] H. P. Breuer, B. Kappler, and F. Petruccione, Phys. Rev. A 59, 1633 (1999).

[26] H. P. Breuer, B. Kappler, and F. Petruccione, Ann. Phys. (N.Y.) 291, 36 (2001).

[27] Y. J. Yan, Phys. Rev. A 58, 2721 (1998); R. X. Xu and Y. J. Yan, J. Chem. Phys. 114, 3868 (2001).

[28] M. Schröder, U. Kleinekathöfer, and M. Schreiber, J. Chem. Phys. 124, 084903 (2006).

[29] E. Ferraro, M. Scala1, R. Migliore, and A. Napoli, Phys. Rev. A 80, 042112 (2009).
[30] F. Shibata, Y. Takahashi, N. Hashitsume, J. Stat. Phys. 17, 171 (1977); S. Chaturvedi and F. Shibata, Z. Phys. B 35, 297 (1979).

[31] U. Kleinekathöfer, J. Chem. Phys. 121, 2505 (2004).

[32] K.-L. Liu and H.-S. Goan, Phys. Rev. A 76, 022312 (2007).

[33] I Sinayskiy et al., J. Phys. A: Math. Theor. 42, 485301 (2009).

[34] D Mogilevtsev et al., J. Phys.: Condens. Matter 21, 055801 (2009)

[35] P. Haikka and S. Maniscalco, Phys. Rev. A 81, 052103 (2010); P. Haikka, arXiv:0911.4600.

[36] Md. M. Ali, P.-W. Chen, and H.-S. Goan, Phys. Rev. A 82, 022103 (2010).

[37] P.-W. Chen, C.-C. Jian and H.-S. Goan, Phys. Rev. B (in press, arXiv:1101.2393).

[38] S. Welack, M. Schreiber and U. Kleinekathofer, J. Chem. Phys. 124044712 (2006).

[39] U. Harbola, M. Esposito, and S. Mukamel, Phys. Rev. B 74, 235309 (2006).

[40] H.-S. Goan, G. J. Milburn, H. M. Wiseman, and H. B. Sun, Phys. Rev. B 63, 125326 (2001);H.-S. Goan and G. J. Milburn, Phys. Rev. B 64, 235307 (2001); H.-S. Goan, Quantum Inf. Comput. 3, 121 (2003); H.-S. Goan, Phys. Rev. B 70, 075305 (2004).

[41] X. Q. Li, W. K. Zhang, P. Cui, J. Shao, Z. Ma and Y. Yan, Phys. Rev. B 69, 085315 (2004). X. Q. Li, P. Cui and Y. Yan, Phys. Rev. Lett 94, 066803 (2005); X. Q. Li, J. Y. Luo, Y. G. Yang, P. Cui and Y. Yan, Phys. Rev. B 71, 205304 (2005); X. Q. Li and Y. Yan, Phys. Rev. B 75, 075114 (2007).

[42] D. W. Utami, H.-S. Goan, and G. J. Milburn, Phys. Rev. B 70, 075303 (2004); D. W. Utami, H.-S. Goan, C. A. Holmes, and G. J. Milburn, Phys. Rev. B 74, 014303 (2006); J. Twamley, D. W. Utami, H.-S. Goan and G. Milburn, New J. Phys. 8, 63 (2006).

[43] P. Zedler, G. Schaller, G. Kiesslich, C. Emary and T. Brandes, Phys. Rev. B, 80045309 (2009).

[44] V. Moldoveanu, A. Manolescu, and V. Gudmundsson, New J. Phys. 11, 073019 (2009); V. Gudmundsson, C. Gainar, C.-S. Tang, V. Moldoveanu, and A. Manolescu, New J. Phys. 11, 113007 (2009).

[45] W. Y. T. Matisse and W. M. Zhang, Phys. Rev. B, 78 235311(2008); J. Jin,M. W. Y. Tu,W. M. Zhang and Y. Yan, New J. Phys. 12, 083013 (2010); X. Zheng, J. Luo, J. Jin and Y. Yan, J. Chem. Phys. 130, 124508 (2009).

[46] G. W. Semenoff and H. Umezawa, Nucl. Phys. B 220, 196 (1983).

[47] T. Yu, Phys. Rev. A 69, 062107(2004).

[48] L. Diósi, N. Gisin, and W. T. Strunz, Phys. Rev. A 58, 1699 (1998). 